\title{
Bile acids stimulate GLP-1 release by accessing basolateral GPBAR1 (TGR5)
}

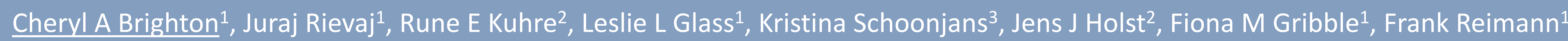

${ }^{1}$ WT-MRC Institute of Metabolic Science, University of Cambridge, UK. ${ }^{2}$ NNF Centre for Basic Metabolic Research, University of Copenhagen, Denmark. ${ }^{3}$ nnstitute of Bioengineering, Ecole Polytechnique Fédérale de Lausanne, Switzerland.

\section{Background}

Glucagon-like peptide 1 (GLP-1) is an incretin hormone released from enteroendocrine L-cells in the gut. GLP-1 analogues and dipeptidyl-peptidase- 4 inhibitors are currently used to treat type-2 diabetes. A greater understanding of the mechanisms underlying the release of GLP-1 may facilitate the development of therapeutics to stimulate the release of endogenous GLP-1. Bile acids have been shown to induce GLP-1 release via the G proteincoupled bile acid receptor 1 (GPBAR1/TGR5) and increased cAMP. The apical sodiumdependent bile acid transporter (ASBT) and nuclear farnesoid $X$ receptor (FXR) may also be involved.

\section{Approach}

Enteroendocrine cells, such as the GLP-1 releasing L-cells, make up less than $1 \%$ of intestinal epithelial cells. The study of these specialised cells is facilitated by transgenic mice expressing fluorescent sensors (Epac2camps or GCaMP3) specifically in L-cells. GLP-1 release was measured from primary murine intestinal cultures and tissue segments mounted in Ussing chambers using a MesoScale Discovery assay.

Aim To identify pathways of bile acid-stimulated GLP-1 secretion and whether these are activated from the apical or basolateral direction.

\section{Conclusion}

Bile acids stimulate GLP-1 secretion primarily via activation of GPBAR1 on the basolateral surface of intestinal L-cells. This suggests the stimulation of gut hormone secretion may include post-absorptive mechanisms. It could impact the design of therapeutics which target GPBAR1 as a means of increasing endogenous gut hormone secretion.

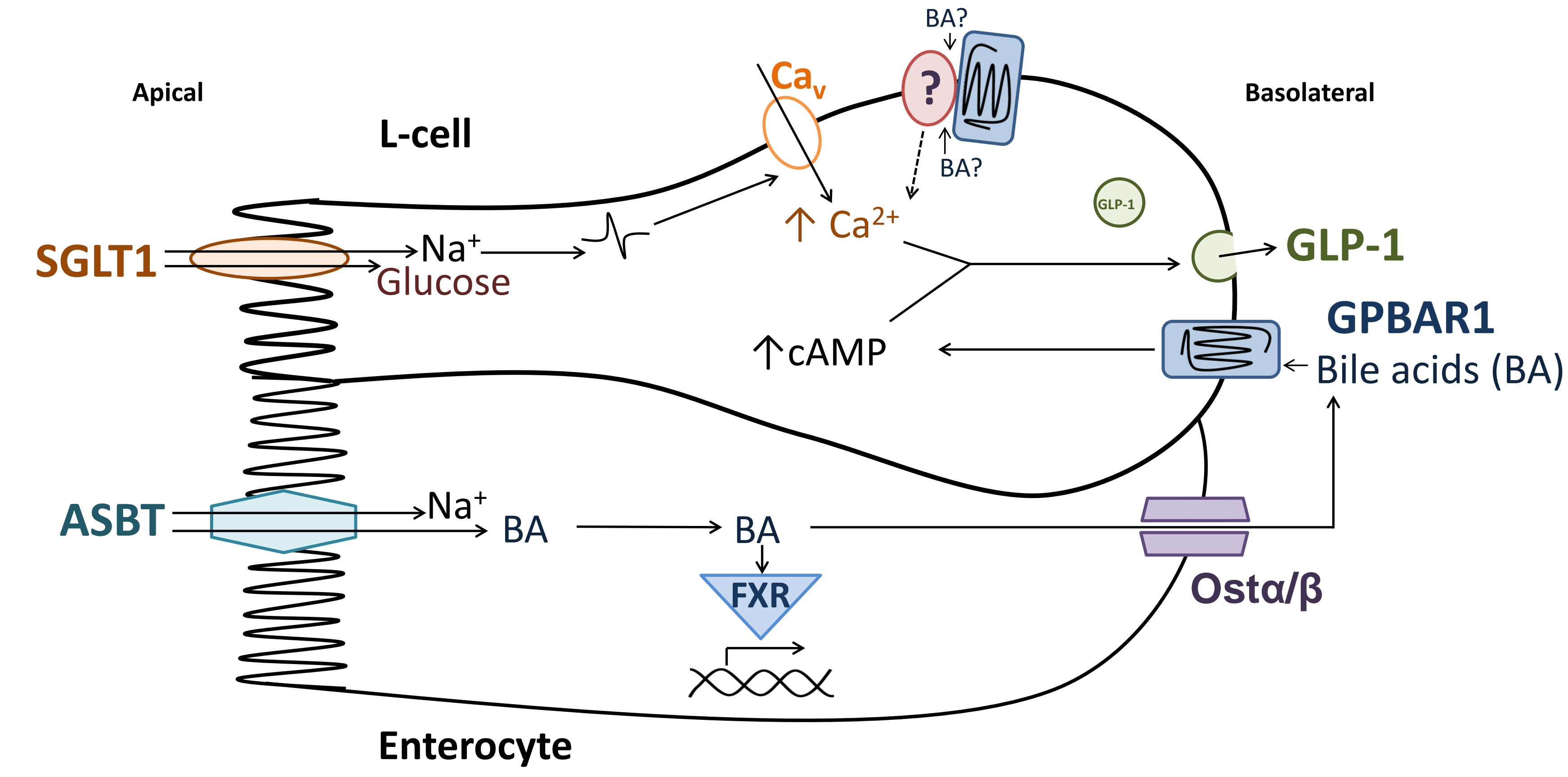

\section{Results}

\section{Bile acid-induced GLP-1 secretion}

GPBAR1 is activated preferentially by TLCA or its specific agonist GPBAR-A (GP-A), however TDCA is a significantly stronger stimulus at $100 \mu \mathrm{M}$.

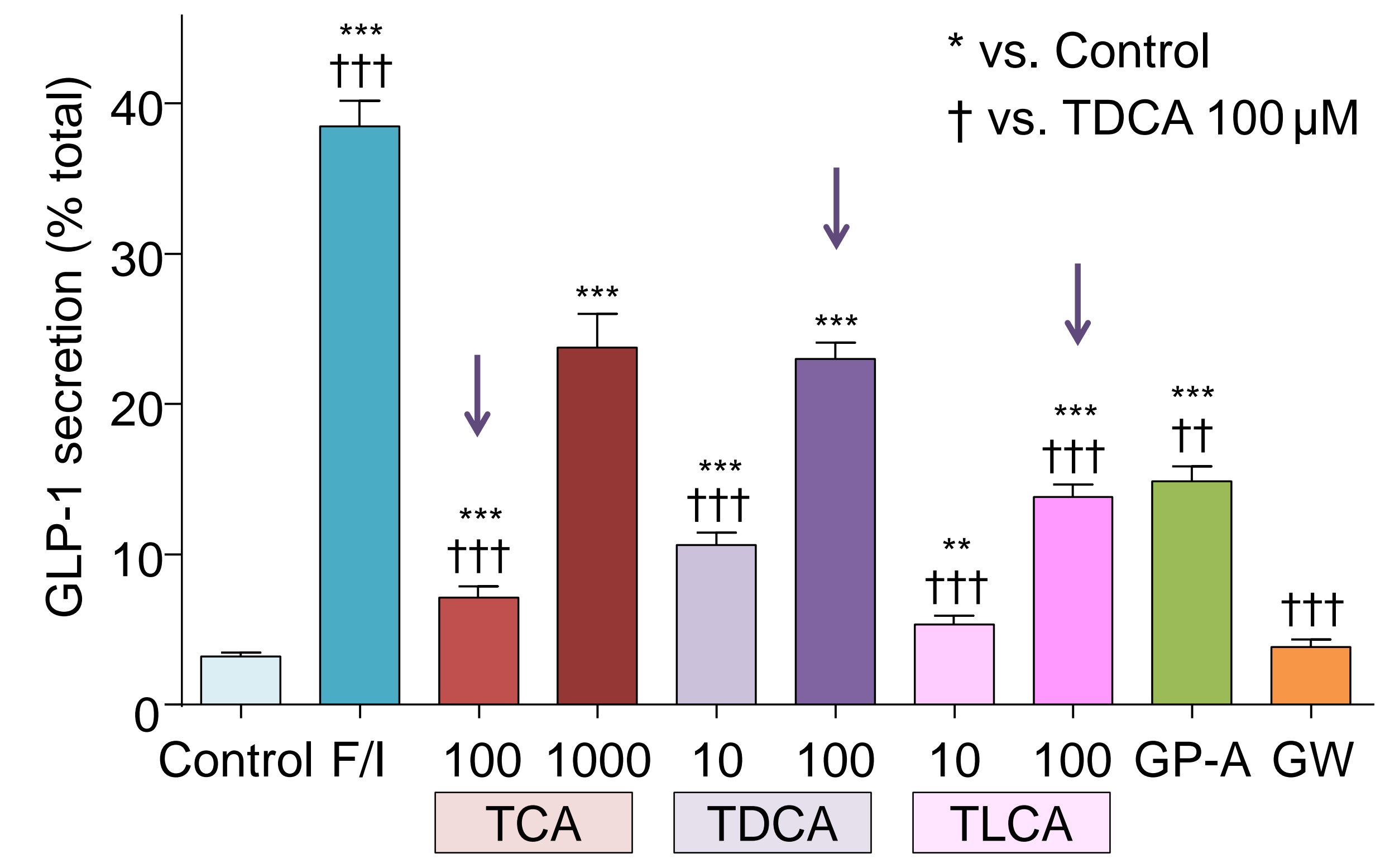

\section{Intracellular cAMP is increased by bile acids}

Measured in intestinal cultures using a FRET-based fluorescent cAMP sensor, Epac2camps, expressed specifically in L-cells.

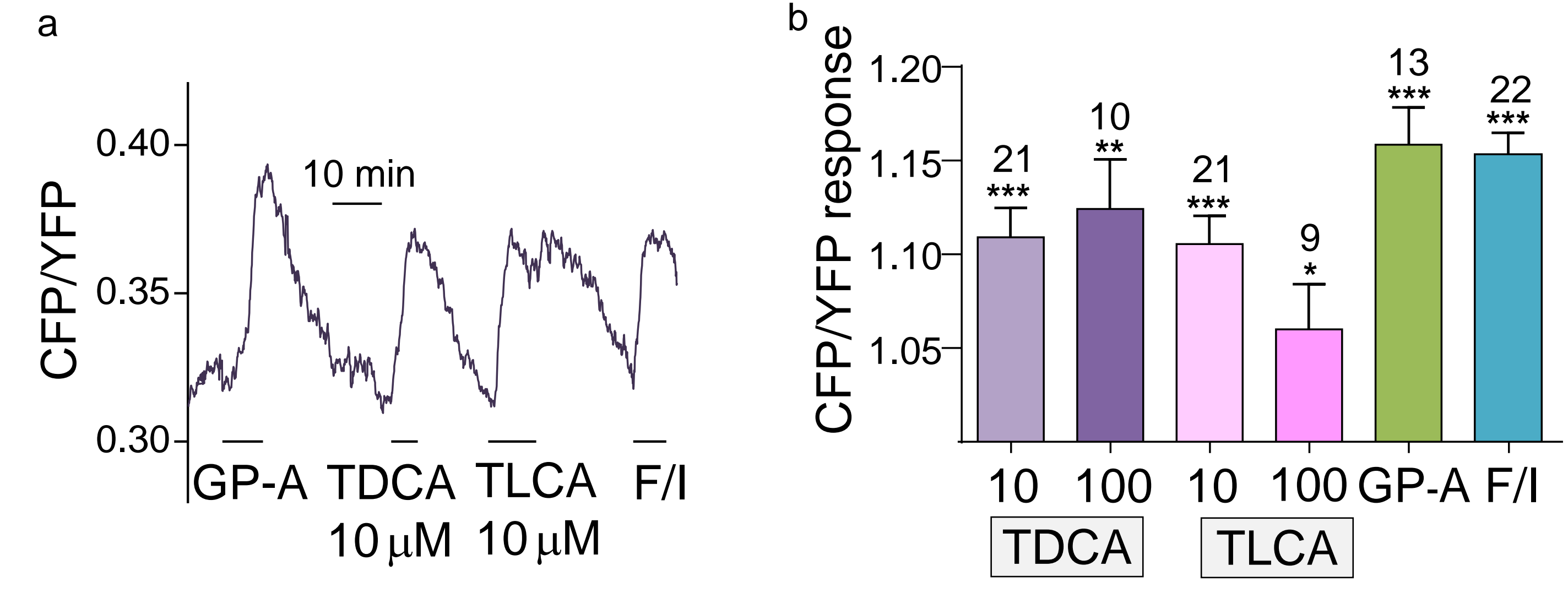

Figure 2 TDCA TLCA

\section{Bile acids stimulate influx of $\mathrm{Ca}^{2+}$}

Measured in intestinal cultures using a fluorescent calcium sensor, GCaMP3, expressed specifically in L-cells.

- TDCA and TLCA trigger rises in intracellular $\mathrm{Ca}^{2+}$

- Specific agonists of GPBAR1 (GP-A) and FXR (GW) produce minimal calcium responses

- Use of $\mathrm{CoCl}_{2}$ indicates $\mathrm{Ca}^{2+}$ enters via voltage gated calcium channels (not shown).

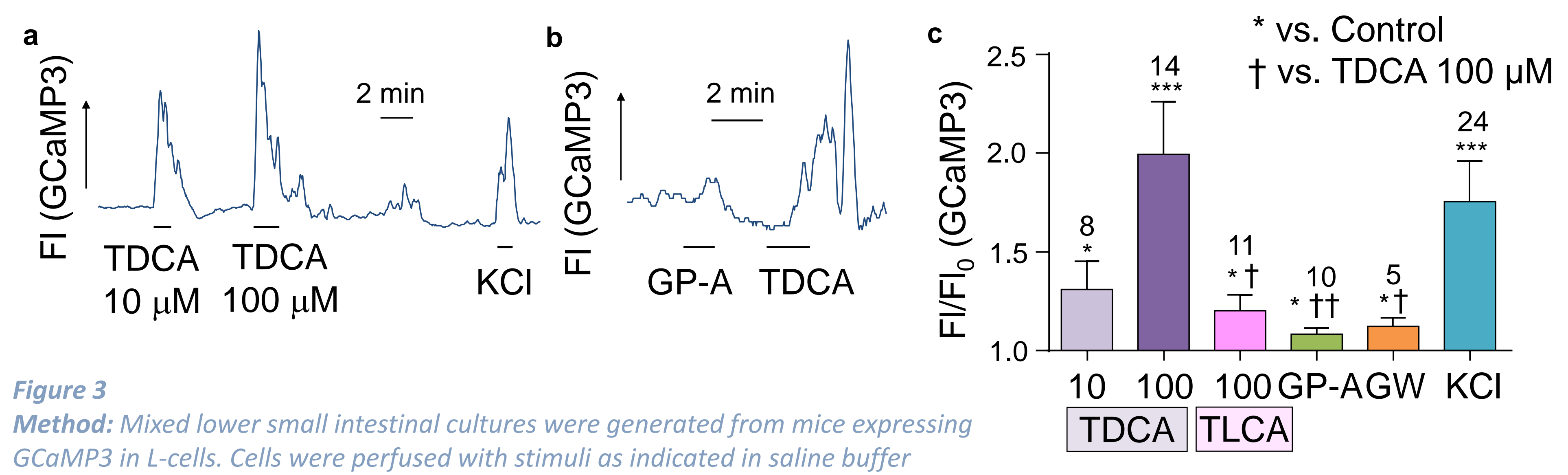

\section{Bile acid uptake via ASBT}

An ASBT-inhibitor (ASBT-I) has no effect upon bile acid induced GLP-1 secretion and intracellular $\mathrm{Ca}^{2+}$ changes in intestinal cultures.
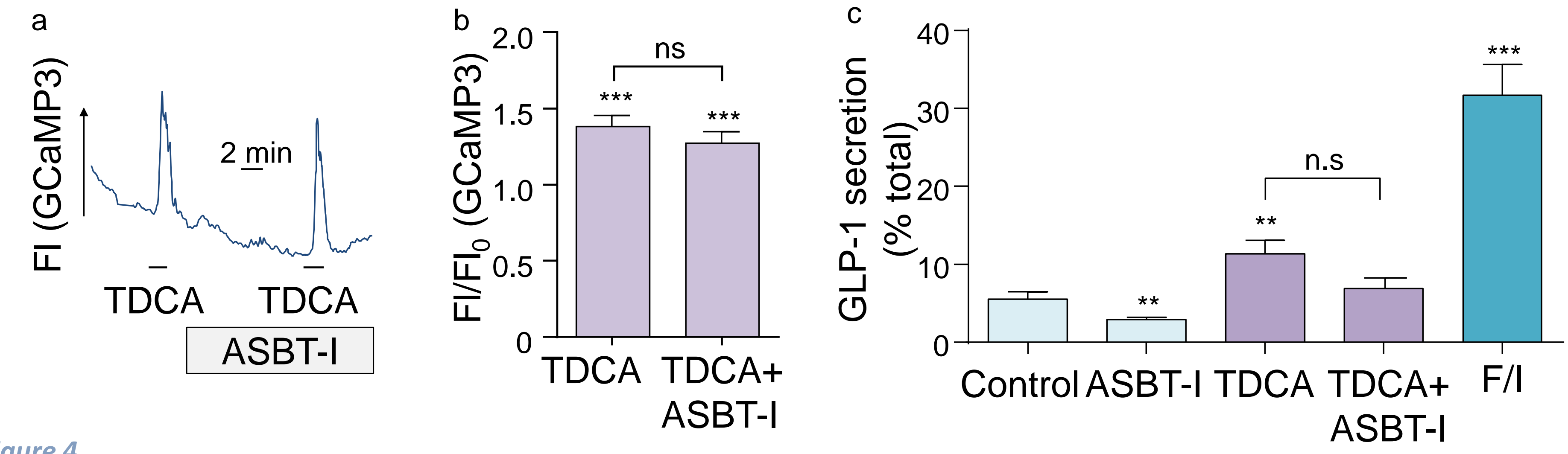

igure 4 ASBT-I

\section{Bile acids stimulate GLP-1 secretion from the basolateral side}

- Basolateral stimulation by bile acids is greater than upon apical

application. Supported by results in perfused rat intestine (not shown).

- Apical stimulation by TDCA is blocked by an ASBT inhibitor.

- A specific GPBAR agonist (GP-A) only stimulates from the

basolateral side.
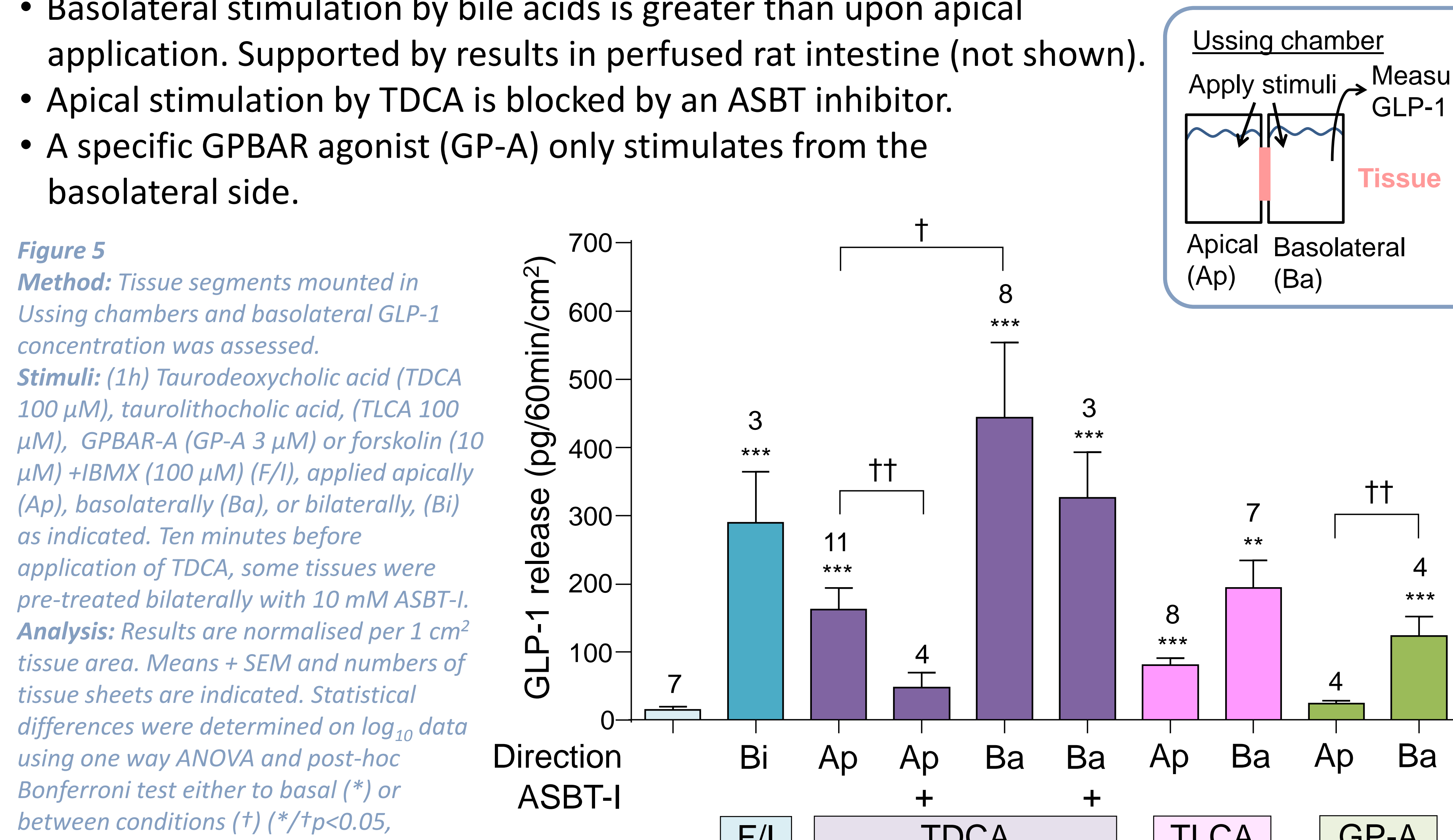

\section{GPBAR1 is essential for bile acid stimulated GLP-1 secretion}

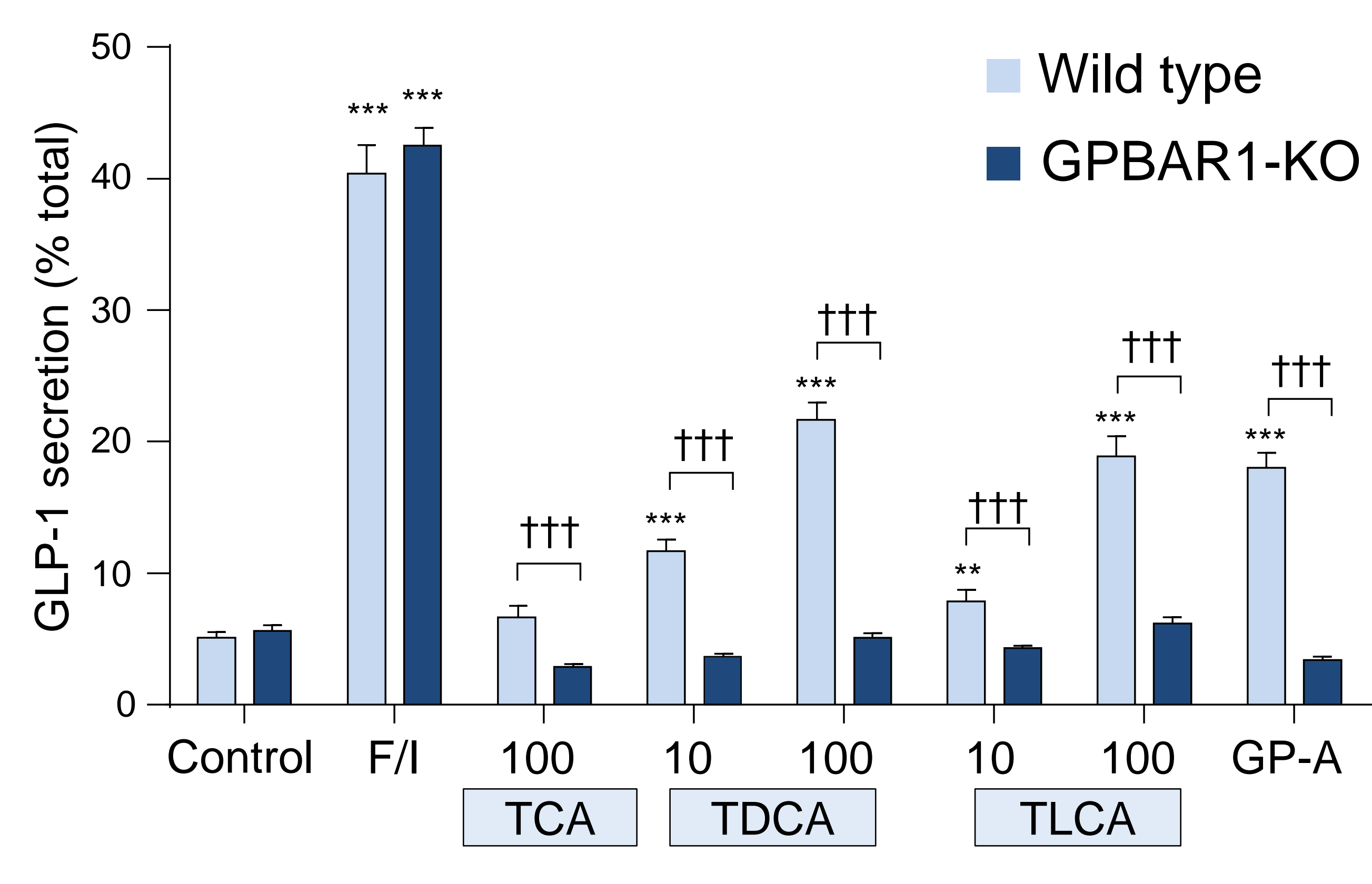

